\title{
Reproducible Research in the era of Next Generation Sequencing: current approaches, examples and future perspectives
}

Claudia Angelini, Dario Righelli, Francesco Russo Istituto per le Applicazioni del Calcolo-CNR Laboratory of Statistics and Computational Tools for Bioinformatics, Napoli, Italy http://bioinfo.na.iac.cnr.it/BioinfoLab/
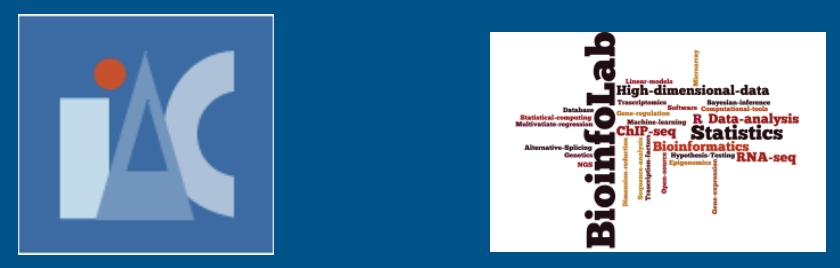


\section{Motivations}

$\square$ The possibility to replicate scientific findings using independent investigators, methods, data, equipment and protocols is the standard approach by which scientific claims are evaluated.

$\square$ In many fields, including bio-medicine and genomics, some studies cannot be fully replicated because of a lack of time or resources (and also since journals often do not publish replicated studies).

$\square$ In such cases, it is important to be able to inspect and reproduce the entire analysis carried out in a given paper.

$\square$ Unfortunately, the description of the data analysis is often lacking of important (technical) details.

$\square$ Moreover, the analyses of NGS "multi-omic" data are also very complex.

$\square$ Therefore, it becomes often very hard to reproduce the results. 


\section{Research Pipeline}

\section{Author}

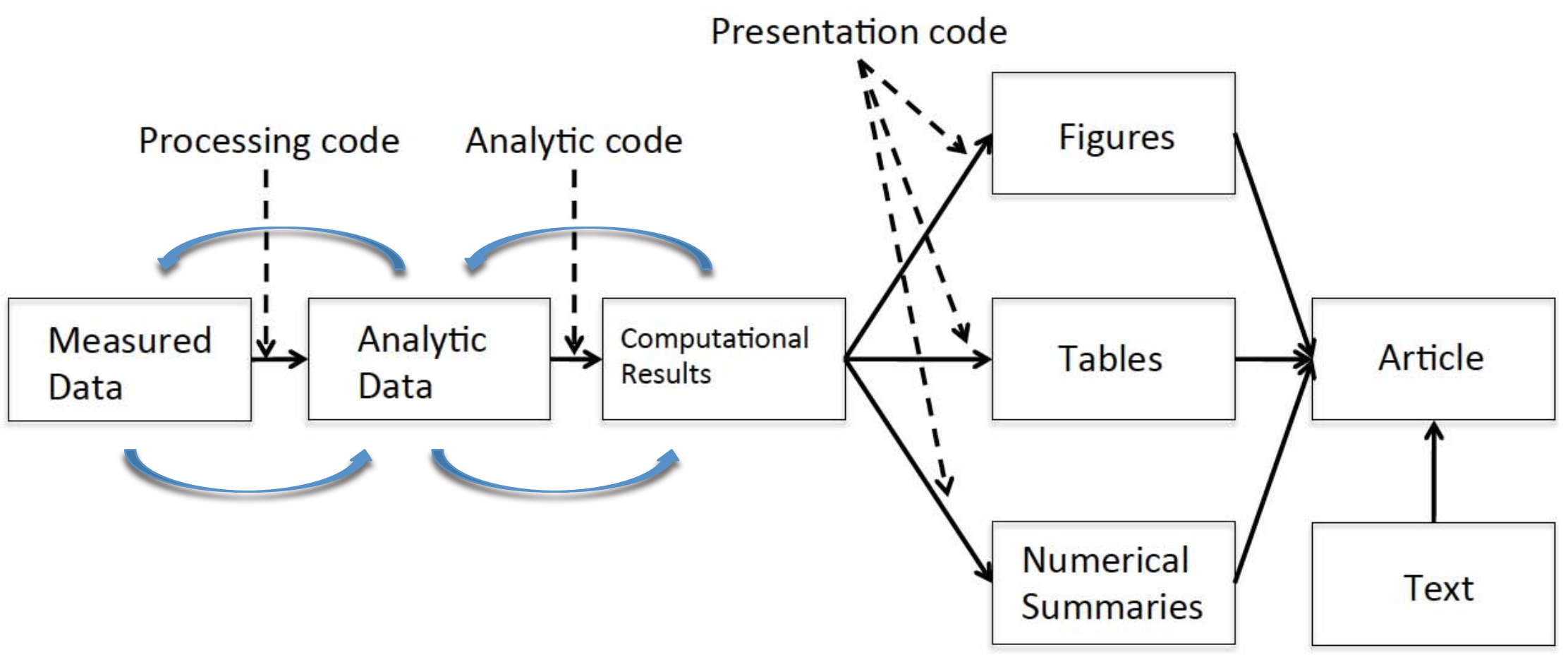

Peng, R., and S. Eckel, "Distributed Reproducible Research Using Cached Computations," 


\section{Reproducible Research}

The idea of $\mathbf{R} \mathbf{R}$ is to make analytic data and code (and its documentation) available so that others may reproduce the findings.

$\square$ From a computational point of view $\mathbf{R} \mathbf{R}$ is similar to regard data analysis as an "experimental protocol".

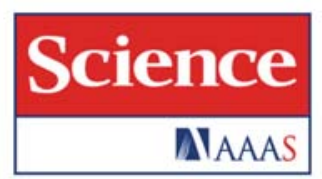

Reproducible Research in Computational Science Roger D. Peng

Science 334, 1226 (2011)

DOI: $10.1126 /$ science. 1213847

Reproducibility Spectrum

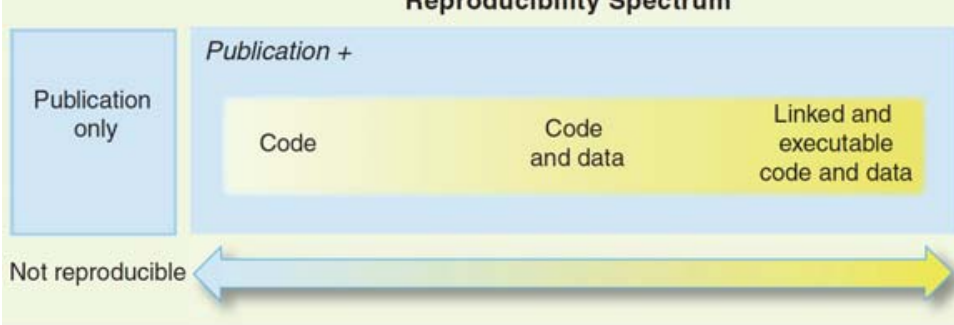

Gold standard

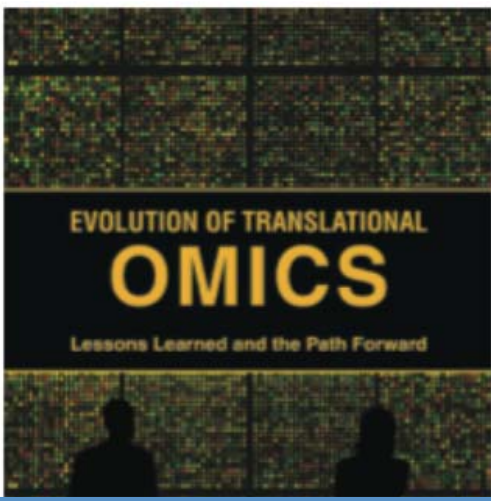

Institute of Medicine

Reports 2012
- Data/metadata used to develop test should be made publicly available

- The computer code and fully specified computational procedures used for development of the omics- data analysis should be made sustainably available

- All aspects of the analysis need to be transparently reported. 


\section{Reproducible Research and NGS}

\section{PERSPECTIVES}

(10)APPLICATIONS OF NEXT-GENERATION SEQUENCING - OPINION

Next-generation sequencing data interpretation: enhancing reproducibility and accessibility

Anton Nekrutenko and James Taylor

Abstract | Areas of life sciences research that were previously distant from each other in ideology, analysis practices and toolkits, such as microbial ecology and personalized medicine, have all embraced techniques that rely on next-generation sequencing instruments. Yet the capacity to generate the data greatly outpaces our ability to analyse it. Existing sequencing technologies are more mature and accessible than the methodologies that are available for individual researchers to move, store, analyse and present data in a fashion that is transparent and reproducible. Here we discuss currently pressing issues with analysis, interpretation, reproducibility and accessibility of these data, and we present promising solutions and venture into potential future developments.
Box 2|Barriers to reproducibility are widespread

Many classical publications in life sciences have become influential because they provide complete information on how to repeat reported analyses so others can adopt these approaches in their own research, such as for chain termination sequencing technology that was developed by Sanger and colleagues ${ }^{35}$ and for PCR ${ }^{36,37}$. Today's publications that include computational analyses are very different. Next-generation sequencing (NGS) technologies are undoubtedly as transformative as DNA sequencing and PCR were more than 30 years ago. As more and more researchers use high-throughput sequencing in their research, they consult other publications for examples of how to carry out computational analyses. Unfortunately, they often find that the extensive informatics component that is required to analyse NGS data makes it much more difficult to repeat studies published today. Note that the lax standards of computational reproducibility are not unique to life sciences; the importance of being able to repeat computational experiments was first brought up in geosciences ${ }^{38}$ and became relevant in life sciences following the establishment of microarray technology and high-throughput sequencing ${ }^{3,39,40}$. Replication of computational experiments requires access to input data sets, source code or binaries of exact versions of software used to carry out the initial analysis (this includes all helper scripts that are used to convert formats, groom data, and so on) and knowing all parameter settings exactly as they were used. In our experience (BOX 1 and Supplementary information S1 (table)), publications rarely provide such a level of detail, making biomedical computational analyses almost irreproducible. Supplementary information S2 (reference list)

lists 50 papers randomly selected from 378 manuscripts published in 2011 that use the BurrowsWheeler Aligner ${ }^{15}$ for mapping Illumina reads. Most papers (31) provide neither a version nor the parameters used, and neither do they provide the exact version of the genomic reference sequence. From the remaining 19 publications, only four studies provide settings, eight studies list the version, and only seven studies list all necessary details. More than half of the studies (26 out of 50) do not provide access to the primary data sets. In two cases, authors provided links to their own websites, where data were deposited; however, in both cases, links were broken.

NGS analyses are quite complex and require the use of several tools Tools are often regularly updated, technology changes continuously NGS analyses are time-consuming and have to handle "Big-data" 


\section{Developing computational tools in the spirit of $\mathbf{R R}$}

One of the goals of modern bioinformatics should be to develop computational tools that support reproducible research

$\square$ Several instruments and have been developed to facilitate $R R$ in different programming languages

$\square \quad \mathbf{R}$ is an open source language, particularly designed for RR. Bioconductor contains several hundreds of packages for the analysis of NGS

Key ingredients for RR are

- Literate statistical programming

- Caching (for handling big data)

- Versioning control

- Suitable result reporting tools and data repositories
Orchestrating high-throughput genomic analysis with Bioconductor

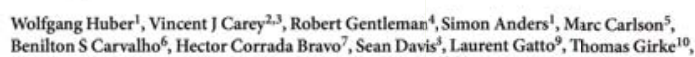

Raphael Gottardo o 11 , Florian Hahne $^{12}$, Kasper D Hansen ${ }^{1,14}$, Rafael A Irizarry $^{3,15}$,

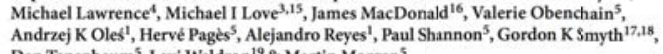

The $R$ Series

Implementing

Reproducible

Research

PERSPECTIVE

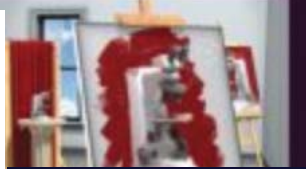

The R Series

Reproducible Research with $\mathbf{R}$ and Rstudio

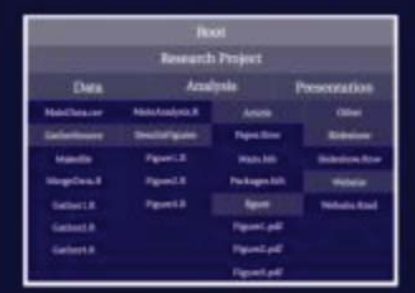

Christopher Gandrud 


\section{Big Data Challenges \& Chaching}

- Cache is a module to store data in order to retrieve that data faster.

$\square \quad$ This helps in RR serving stored data resulting from time consuming chunk of code.

- In this way it avoids repetition of time consuming computation when they are computed again.

- Additionally permits to share cached data through the web.

- In this way it is possible to reproduce the same computations using the same data or to verify the results of a third part computations.

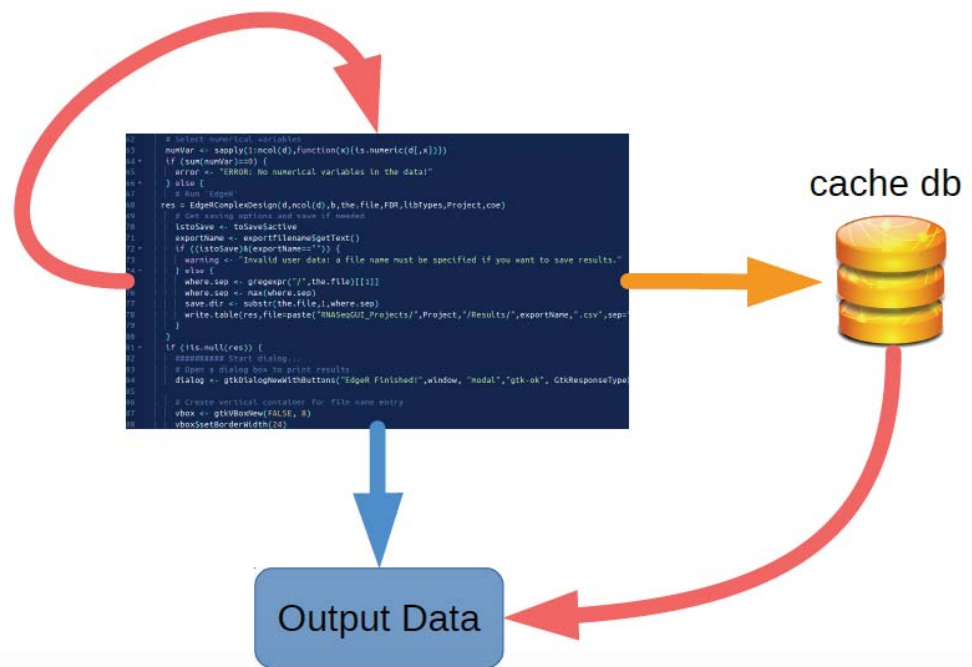




\section{What about GUI?}

$\square$ Many tools for omics data analysis have a graphical user interfaces (GUls)

$\square$ GUls are convenient and very intuitive for biologists.

$\square$ GUls are also interactive, so that the user can decide what kind of analysis to perform (point-and click approach) on the basis of the intermediate results

$\square$ Tools with web-interface have similar features (and share similar issues)

$\square$ However, GUls do not facilitate RR, since results are obtained after clicking several buttons $\rightarrow$ difficult to keep track of all performed steps

Our Aim: To develop user friendly computational tools for NGS data analysis in the spirit of "Reproducible Research", i.e., we want combine $\mathbf{G U I}$ with tools for $\boldsymbol{R} R$ available in $R$. 


\section{RNASeqGUI}

$\square$ RNASeqGUl is implemented in $\mathbf{R}$. It requires the RGTK2 graphical Library to run

It uses BiocParallel to speed up the computations.

\section{RNASeqGUI can be downloaded from http://bioinfo.na.iac.cnr.it/RNASeqGUI/}

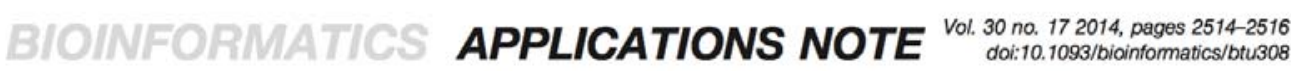

Gene expression

Advance Access publication May 7, 2014

RNASeqGUI: a GUI for analysing RNA-Seq data

Francesco Russo* and Claudia Angelini

Istituto per le Applicazoni del Calcolo, CNR, 80131, Napoli, Italy

Associate Editor: No Hofacker

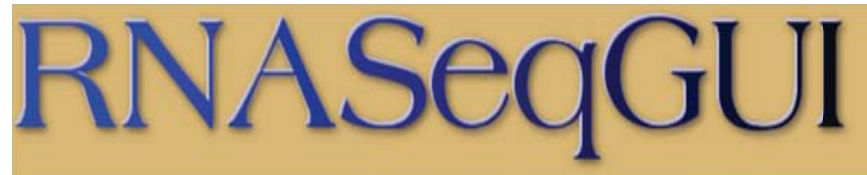

Home Example Manual Download Contact Material Credits

A GUI for the identification of differentially expressed genes that supports Reproducible Research.

Authors: Dr Francesco Russo and Dr Claudia Angelini (IAC-CNR) Additionally, Dario Righelli is collaborating to the development of RNASeqGUI since version CNR 0.99 .3

Last update (version 0.99.4) March 11, 2015 IAC

RNASeqGUI R package is a graphical user interface for the identification of differentially expressed genes from RNA-Seq experiments.

RNASeqGUl is implemented in R following and expanding the idea presented in tuxette-chix.

RNASeqGUl includes several well known RNA-Seq tools, available as command line in Bloconductor.

RNASeqGUI is divided into seven main sections. Each section is dedicated to a particular step of the data analysis process. The first section covers the exploration of the bam files. The second concerns the counting process of the mapped reads against a genes annotation file. The third focuses on the exploration of count-data, on the normalization procedures and on the filtering process. The fourth is about the identification of the differentially expressed genes that can be performed by several methods, such as: EdgeR Exact Test, EdgeR GLM, DESeq, DESeqComplexDesign, DESeq2, DESeq2ComplexDesign, NoiSeq, BaySeq. The fifth section regards the inspection of the results produced by these methods and the quantitative comparison among them. The six seation reanards the Gene-Set and Pathwav analusis. 


\section{RNASeqGUI workflow}

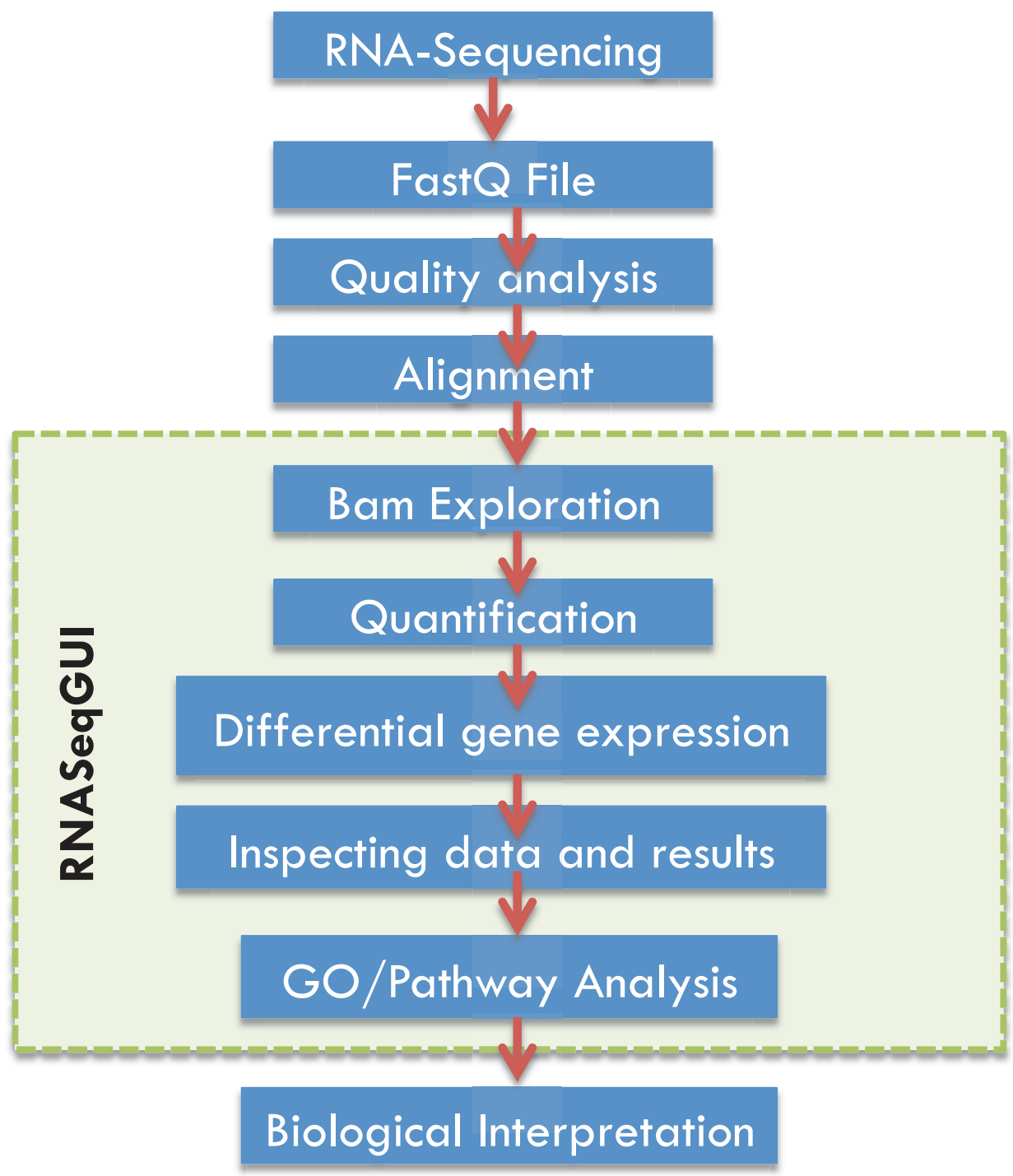

Recent update includes

$\square$ It handles technical and biological replicates

$\square$ Complex experimental designs in differential gene expression section

$\square$ Filtering and Conversion

$\square$ Pathway analysis using Gage and Graphite

$\square$ Fancy reporting using Reportingtools

$\square$ Advanced RR using $\mathbf{R}$ markdown and knitr

$\square$ Caching using filehash 


\section{RNASeqGUI Main Interface}

BAM EXPLORATION SECTION

Bam Exploration Interface

COUNT SECTION

Read Count Interface

PRE-ANALYSIS SECTION

Data Exploration Interface

Normalization Interface

Eiltering Interface

DATA ANALYSIS SECTION

Data Analysis Interface

POST ANALYSIS SECTION

Besult Inspection Interface

Result Comparison Interface

GENE-SET/PATHWAY SECTION

Graphite Interface

Gage Interface

REPORT AND UTILITY SECTION
The GUI is divided into several sections. Each section is dedicated to a particular step of the data analysis process.

The analysis starts by creating a project or opening an existing project.

Then, the user can access any of RNASeqGUl sections.

Data Analysis Section is the core of RNASeqGUI and contains several methods to identify differentially expressed genes (DE). 


\section{Analyzing data with RNASeqGUI}

$\square$ BAM Exploration Section

$\square$ Count Section

$\square$ Pre-Analysis Section

$\square$ Data Analysis Section

$\square$ Post-Analysis Section

$\square$ GeneSet/pathway Section

$\square$ Report and Utility Section

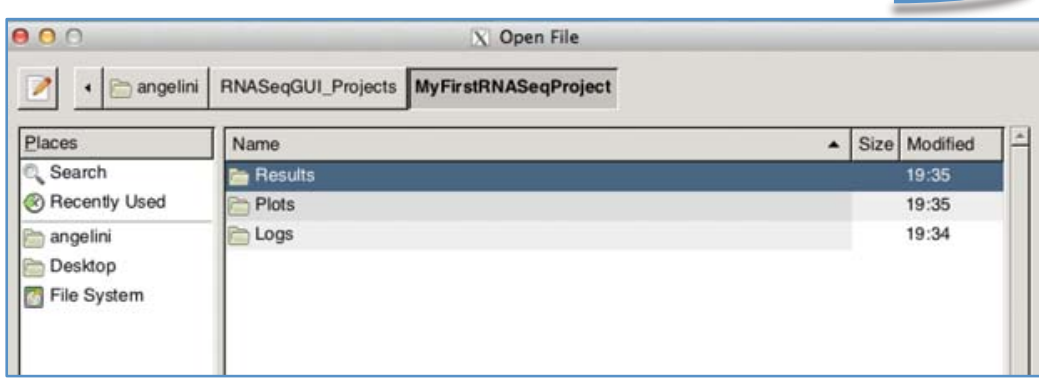

$\square$ Results are given in terms of tabdelimited-files, user friendly htmlpages, summary tables, and figures $\rightarrow$ Folder Result

$\square$ Figures are in pdf, and are customizable (in terms of colors and scale) $\rightarrow$ Folder Plots

$\square$ All actions are stored in a html report and $\mathbf{R m d}$ re-executble code $\rightarrow$ Folder Logs

Moreover, thanks to caching, data, intermediate and final results are directly connected with databases.

$\square$ Reporting tools provide navigable results directly linked to Ensembl 


\section{RR in RNASeqGUI (1)}

The spirit of RR is now fully incorporated in RNASeqGUI.

$\square$ Thanks to the use of $R$ markdown language, RNASeqGUI automatically generates a dynamic report of all analysis carried out on a given Project. The report includes both the $R$ code, the figures and the summary of the results. The report can be executed and results are being updated automatically, if changes occur. The report also includes all versions of the $R$ packages used, all steps, input/output parameters, file names and so on.

$\square$ The report can be exported as HTML

$\square$ Caching is used to speed up repetitive and computational expensive function calls by using results stored in pre-computed data-bases

$\square$ The report and the cached database are suitable for being submitted as documentary $R$ file of data analysis for $R R$ publication 


\section{RR in RNASeqGUI (2)}

The Report contains "live" $R$ code. Therefore, expert users can use RNASeqGUI to build the skeleton of their pipeline, then they can modify the code, or add their favorite method.

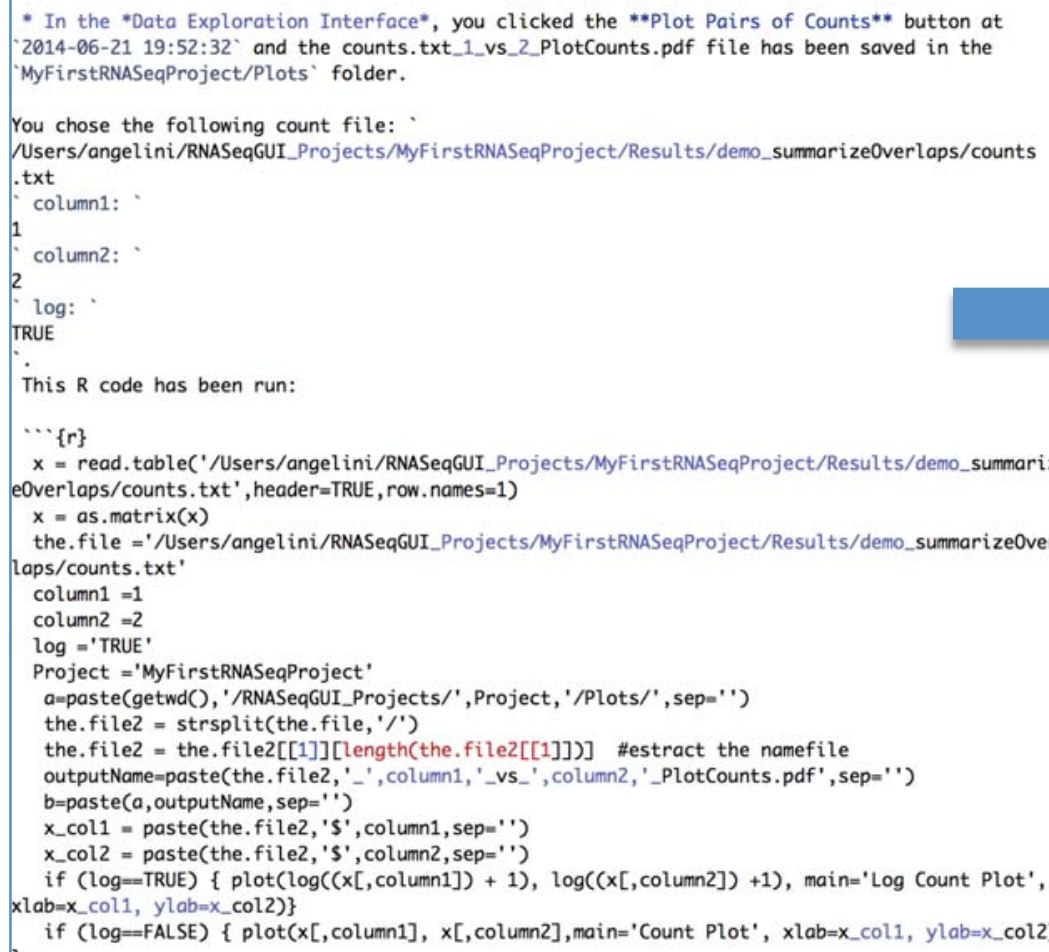

- In the Data Exploration Intertace, you clicked the Plot Pairs of Counts button at 2014-06-21 19:52:32 and the counts.txt_1_vs_2_PlotCounts.pdt file has been saved in the Myfirstras seqproject/Plots folder.

You chose the following count fle:

Jusers/angelini/Rusequi_Projects/MyFirstrasequproject/Results/demo_sumarizeoverlaps/Counts. txt column 1: 1 column2: 2 $\log$ : TRUE. This $\mathrm{A}$ code has been run:

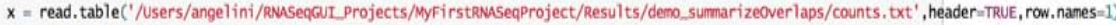
$x=$ as.matrix $(x)$

the. file ='/Users/angel ini/Rusegaut_projects/MyFirstRnasegproject/Results/demo_sumarizeoverlaps/counts. txt'

column $=1$

column $2=2$

$\log =$ 'TRUE'

Project ='MyFirstraseaproject'

a-paste (getwd O, '/RMSeqGuL_Projects/', Project, '/Plots/', sep=" ")

the. file2 $=$ strsplit (the.file, $\left.{ }^{\prime} /{ }^{\prime}\right)$

the.file2 = the.file2[[1]][length (the,file2[[1]])] \#estract the nanofile

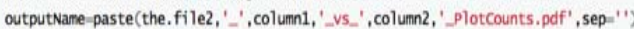
b-paste (a, outputvane, sep - "')

x.col1 = paste(the. file2, 's', column, sep=" ")

$x$ col2 $=$ paste (the. filee, 's', column2, sep ="

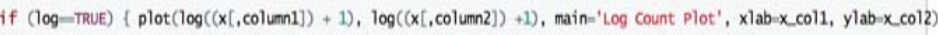
if (log-FALSE) \{ plot(x[,column1], x[, column2], mainn' 'Count Plot', xlab-x_col1, ylab-x_col2) \}

abline $(\mathrm{a}=0, \mathrm{~b}=1, \mathrm{col}=2$

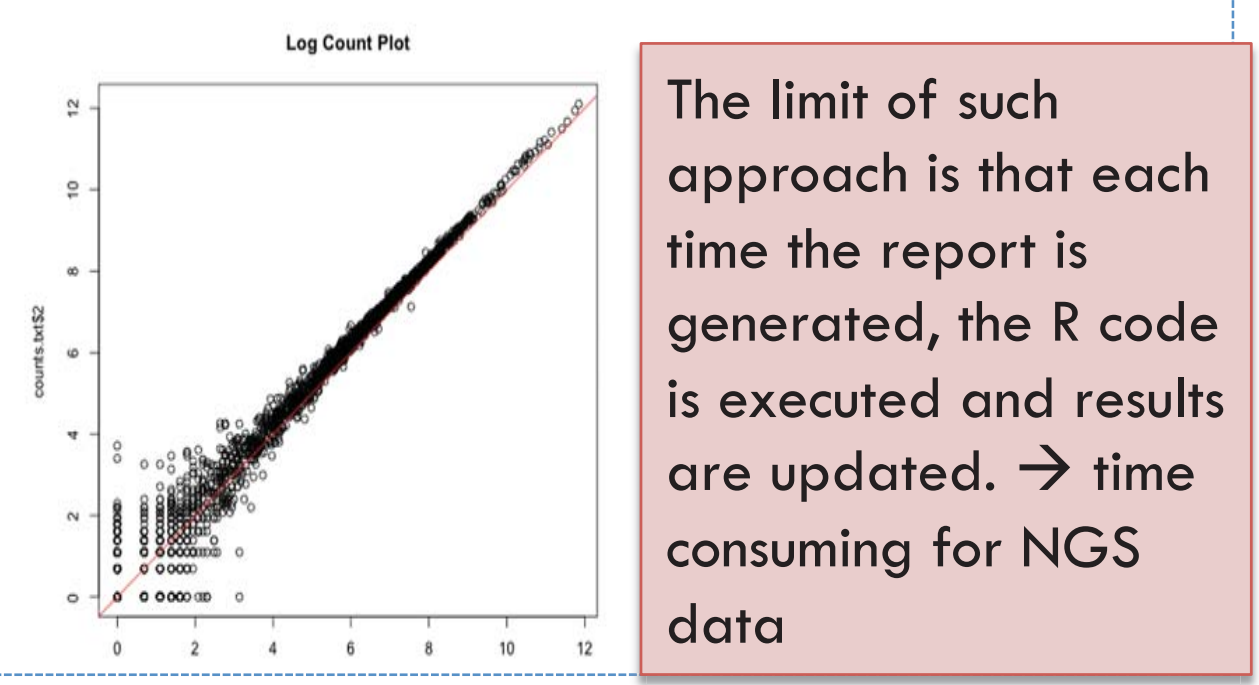




\section{Conclusions}

$\mathbf{R} \mathbf{R}$ is very important for producing good Science, and it is expected that in the near future it will be mandatory.

$\square$ Editors have to encourage and promote RR.

$\square$ For those who develop computational tools it is important to provide novel software able to meet the need of RR.

$\square$ RNAseqGUI is one example in such directions, that combine the GUls with the tools in R for RR. Therefore, for each project RNAseqGUI generates a report that keep track of all actions the user carried out. Moreover all data, intermediate and final results are cached to speed up computation, reporting layout is ameliorated and connected to database and webserver.

Take home message: As potential authors, try the best to produce $R R$, regardless to tool you are using. As potential developer, try the best to release tools that support RR. 
Thanks to all members of
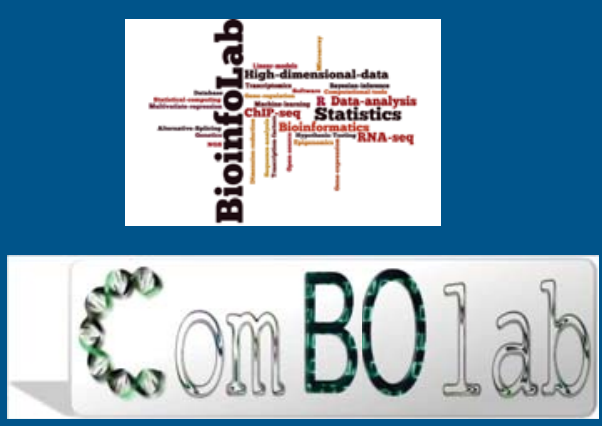

Supporting Projects
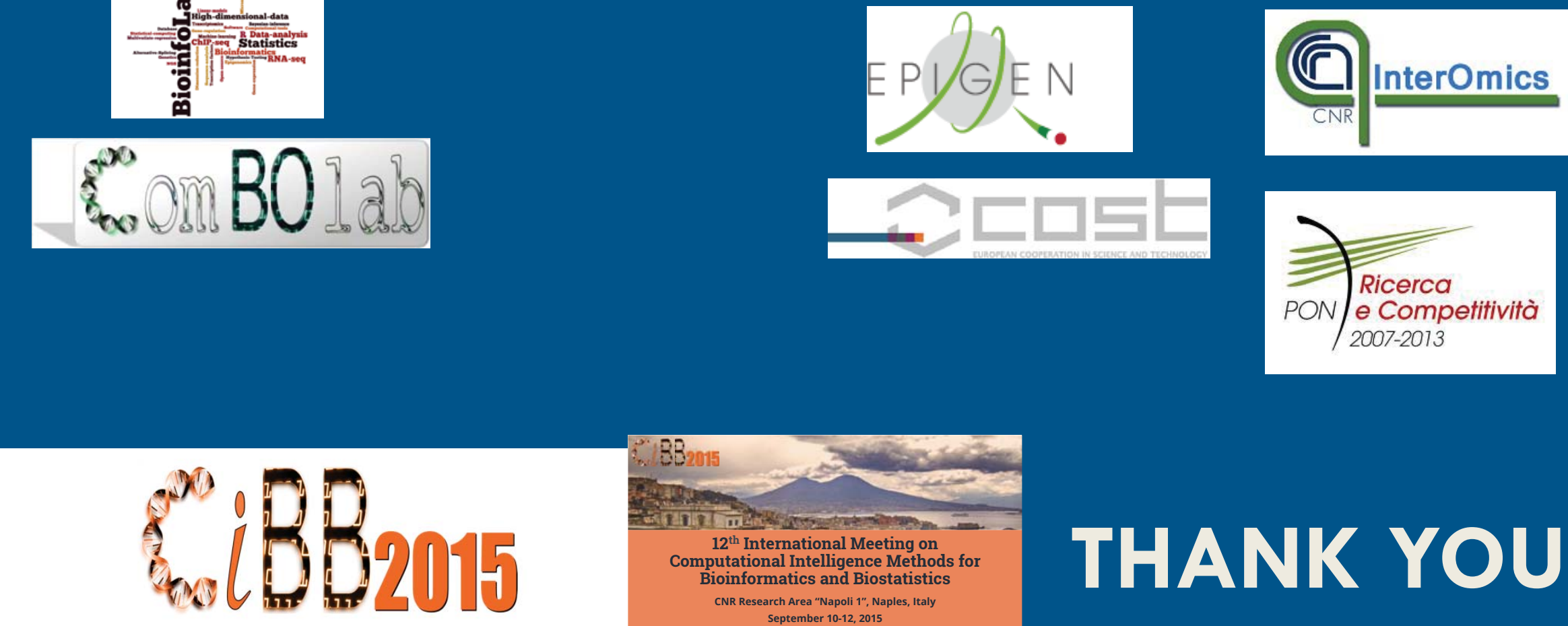

10-12 September 2015, Naples, ITALY.

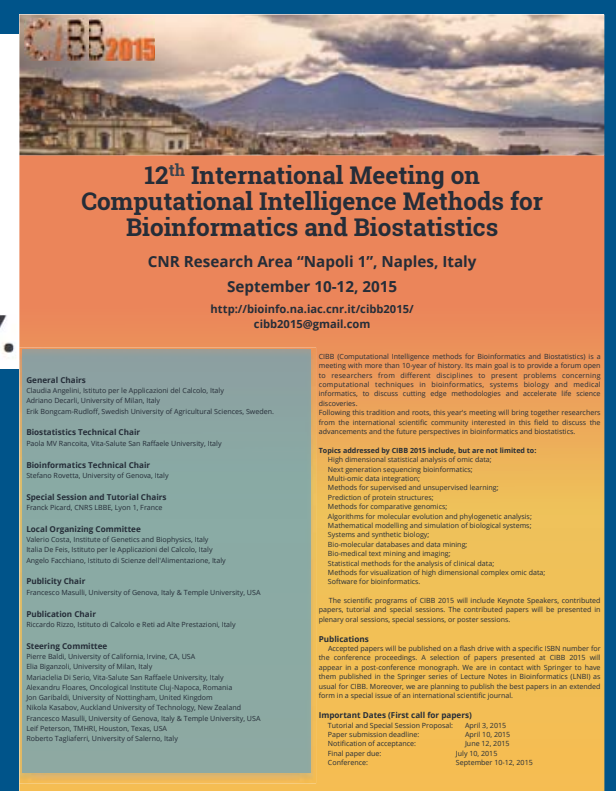

THANK YOU

FOR THE ATTENTION 